\author{
Joseph M. Powers \\ e-mail: powers@nd.edu \\ Associate Professor, \\ Department of Aerospace and Mechanical \\ Engineering, \\ University of Notre Dame, \\ Notre Dame, Indiana 46556-5637
}

\section{On the Necessity of Positive Semi-Definite Conductivity and Onsager Reciprocity in Modeling Heat Conduction in Anisotropic Media}

It is demonstrated by a concise standard derivation, motivated by principles of rational continuum mechanics and irreversible thermodynamics augmented by novel detailed examples, that for heat conduction in linearly anisotropic solids: (1) common restrictions placed on the form of the thermal conductivity tensor are insufficient to guarantee satisfaction of the second law of thermodynamics, and (2) satisfaction of the first and second laws of thermodynamics alone is still insufficient to insure agreement between heat flow predictions and observation. An additional constraint beyond that given in many standard studies, namely that all three principal invariants of the conductivity tensor be positive semi-definite, is imposed in order to guarantee satisfaction of the entropy inequality. Thus constrained, such a theory remains under-restricted and can admit purely cyclic heat fluxes, which are not observed in nature. Imposition of the conjectures of Duhamel and Stokes, which are in fact earlier specific incarnations of Onsager's reciprocity theory, on the constitutive model relating heat flux to temperature gradient is a sufficient remedy. [DOI: $10.1115 / 1.1798913]$

Keywords: Analytical, Conduction, Heat Transfer, Second Law, Thermodynamics

\section{Introduction}

In this work, an exposition is given, accompanied by new illustrative examples and minor clarifications to some of the existing literature, to demonstrate that for energy diffusion in linearly anisotropic solids: (1) restrictions given in common heat transfer references are necessary but insufficient to guarantee satisfaction of the second law of thermodynamics, and (2) fully satisfied first and second laws of thermodynamics provide necessary but insufficient restrictions on the functional forms of common constitutive laws. An additional independent condition, such as that provided by Onsager reciprocity [1], which itself is a generalization of the earlier conjectures of Duhamel [2] and Stokes [3], is required to bring theory into agreement with experiment [4-6] for conduction in an anisotropic medium.

General discussions of issues relevant to Onsager reciprocity and anisotropic heat conduction are common in the communities of irreversible thermodynamics, cf. [7-12]; continuum mechanics, cf. [13-15]; and theoretical physics and statistical mechanics, cf. [16-19]. It is noted, moreover, that statistical mechanics gives an effective microscale based theory which casts macro-scale Onsager reciprocity and the second law of thermodynamics on a more fundamental theoretical foundation. Nevertheless, while often wide ranging, compact statements of theoretical restrictions on models of anisotropic heat conduction are often difficult to pinpoint in these sources; the useful [14] is the most relevant. The discussion in the traditional heat transfer community, cf. [20-31], is often more limited, and, as will be seen, incomplete. Understanding such issues can be of aid to anyone doing computational modeling of anisotropic materials, especially on nonorthogonal grids, designing experiments on materials with anisotropic microstructure, or wanting to build a strong classical foundation from

Contributed by the Heat Transfer Division for publication in the JOURNAL OF HEAT TRANSFER. Manuscript received by the Heat Transfer Division January 29, 2004; revision received June 3, 2004. Associate Editor: R. Pitchumani. which to explore the implications of molecular dynamics on energy diffusion, especially relevant for energy transport on molecular lattice scales in solids.

In what follows, a simple model of diffusive energy transport in an anisotropic solid will be presented. The analysis is restricted to a classical, axiomatic, macro-scale approach with no detailed appeal to the underlying micro-scale physics; however, it is noted that the axioms employed here are consistent with conclusions which can be drawn from micro-scale models. For such a material, a general form of the first law of thermodynamics is posed along with constitutive models for internal energy and diffusive heat flux. A first set of restrictions on the form of the constitutive laws is found by a standard application of the second law of thermodynamics. It will then be shown that these necessary limitations alone nevertheless admit behavior which is not observed in experiment; this is remedied by further application of Onsager reciprocity.

How these principles may be unintentionally violated is then illustrated in three simple examples, which should be useful for pedagogical purposes. First, an anisotropic material which has a purely symmetric thermal conductivity tensor which also satisfies the limited second law requirements of [21], but has a third principal invariant with negative value, in fact violates the second law. Next, for an anisotropic material that has a purely antisymmetric thermal conductivity tensor, it will be shown that a temperature gradient in a given direction induces a heat flux in an orthogonal direction, all the while satisfying the first and second laws. This is demonstrated in two-dimensional geometries which are plane Cartesian and plane polar. In the plane polar geometry, it is seen that purely radial temperature gradients induce purely circumferential heat fluxes. A qualitative analogy is made between such a flux and (1) solid body rotation and (2) the so-called cyclic chemical reactions studied in the original work of Onsager. 


\section{Model and Analysis}

Consider the first law of thermodynamics for an immobile, incompressible solid, posed in Cartesian index notation

$$
\rho \frac{\partial e}{\partial t}=-\frac{\partial q_{i}}{\partial x_{i}}
$$

Here, the standard Einstein convention, in which the repetition of the index $i$ connotes a summation from $i=1$ to 3 , is employed. The independent variables are distance $x_{i}$, where $i=1,2,3$, for each of the orthogonal directions in space, and time $t$. The dependent variables are internal energy per unit mass, $e$, and the heat flux vector, $q_{i}$. The density, $\rho$, is a constant parameter. For the incompressible immobile solid, which can undergo no deformation work process, classical thermodynamics provides a Gibbs equation for systems near equilibrium undergoing reversible heat transfer

$$
d e=T d s,
$$

where $T$ is the temperature and $s$ is the entropy per unit mass. It is then postulated that such a relation is valid for more general systems which may be undergoing irreversible heat transfer. Consequently, one adopts the non-equilibrium $\partial e / \partial t=T \partial s / \partial t$, and the first law, Eq. (1), can be rewritten in terms of entropy as

$$
\rho T \frac{\partial s}{\partial t}=-\frac{\partial q_{i}}{\partial x_{i}}
$$

Next, use the product rule to expand the first law, Eq. (3), in a nonintuitive fashion to get

$$
\rho \frac{\partial s}{\partial t}=\underbrace{-\frac{\partial}{\partial x_{i}}\left(\frac{q_{i}}{T}\right)}_{\text {reversible }}-\underbrace{\frac{q_{i}}{T^{2}} \frac{\partial T}{\partial x_{i}}}_{\text {irreversible }}
$$

This formulation of the first law is written so as to segregate entropy generating terms into those associated with both reversible and irreversible heat transfer. This is seen upon consideration of the second law of thermodynamics, which for such a material is given by

$$
\rho \frac{\partial s}{\partial t} \geqslant-\frac{\partial}{\partial x_{i}}\left(\frac{q_{i}}{T}\right)
$$

Using Eq. (4) to eliminate the terms involving entropy and reversible heat transfer, the second law, Eq. (5), can be re-expressed as a Clausius-Duhem inequality

$$
-\frac{q_{i}}{T^{2}} \frac{\partial T}{\partial x_{i}} \geqslant 0 .
$$

The only term which contributes to the Clausius-Duhem inequality is that associated with irreversible heat transfer. While reversible heat transfer does induce entropy changes, it makes no contribution to the irreversibility of the process.

Next two simple linear constitutive models are posed. First, the internal energy is taken to be a linear function of temperature so that the caloric state equation is

$$
e=c T+e_{o}
$$

where $c$ is the constant specific heat and $e_{o}$ is a reference energy. Second, the heat flux vector is given by Duhamel's generalization [2] of Fourier's law, i.e., it is taken to be a linear function of temperature gradient which vanishes when there is no temperature gradient. Thus,

$$
q_{i}=-k_{i j} \frac{\partial T}{\partial x_{j}}
$$

where $k_{i j}$ is taken to be a constant nonisotropic asymmetric tensor of thermal conductivity. The asymmetric $k_{i j}$ can be decomposed as $k_{i j}=k_{(i j)}+k_{[i j]}$, where the symmetric $k_{(i j)}$ and antisymmetric $k_{[i j]}$ are defined as

$$
k_{(i j)} \equiv k_{(j i)}=\frac{1}{2}\left(k_{i j}+k_{j i}\right), \quad k_{[i j]} \equiv-k_{[j i]}=\frac{1}{2}\left(k_{i j}-k_{j i}\right)
$$

With Eq. (9), Eq. (8) can be recast as

$$
q_{i}=-k_{(i j)} \frac{\partial T}{\partial x_{j}}-k_{[i j]} \frac{\partial T}{\partial x_{j}}
$$

Substituting Eq. (10) into the Clausius-Duhem relation (6), it is seen that the second law for this material becomes, after elimination of the nonzero temperature

$$
k_{(i j)} \frac{\partial T}{\partial x_{j}} \frac{\partial T}{\partial x_{i}}+\underbrace{k_{[i j]} \frac{\partial T}{\partial x_{j}} \frac{\partial T}{\partial x_{i}}}_{=0} \geqslant 0
$$

Now the part of Eq. (11) involving $k_{[i j]}$ is identically zero for arbitrary values of temperature gradient and $k_{[i j]}$. This is because the tensor inner product of a symmetric tensor, such as $\left(\partial T / \partial x_{j}\right)\left(\partial T / \partial x_{i}\right)$ and an antisymmetric tensor, such as $k_{[i j]}$, always vanishes, which can be shown by direct expansion. So the second law reduces to

$$
k_{(i j)} \frac{\partial T}{\partial x_{j}} \frac{\partial T}{\partial x_{i}} \geqslant 0
$$

which must hold for any value of temperature gradient. So it is seen that any nonzero antisymmetric component of $k_{i j}$ cannot influence the evolution of entropy or the second law.

Using standard results from linear algebra, cf. Strang [32], it can further be stated that the entropy inequality, Eq. (12), will be satisfied if and only if $k_{(i j)}$ is positive semi-definite. This will be the case if and only if the eigenvalues $\lambda$ of $k_{(i j)}$ are positive semi-definite. The eigenvalues are guaranteed to be real by the symmetry of $k_{(i j)}$. The eigenvalues of $k_{(i j)}$ can be shown to be positive semi-definite if and only if the so-called invariants of $k_{(i j)}, I_{1}, I_{2}$, and $I_{3}$, are themselves positive semi-definite, which can be inferred from Fung [13]. The invariants reside in the characteristic polynomial of $k_{(i j)}$, which determines the eigenvalues $\lambda$

$$
\lambda^{3}-I_{1} \lambda^{2}+I_{2} \lambda-I_{3}=0
$$

The three roots to Eq. (13) will be denoted as $\lambda_{1}, \lambda_{2}$, and $\lambda_{3}$. Detailed analysis shows that the invariants, along with the necessary and sufficient conditions for positive semi-definiteness of symmetric matrices, are compactly summarized as

$$
\begin{gathered}
I_{1}=k_{(i i)}=\operatorname{tr}\left(k_{(i j)}\right)=\lambda_{1}+\lambda_{2}+\lambda_{3} \geqslant 0 \\
I_{2}=\frac{1}{2}\left(k_{(i i)} k_{(j j)}-k_{(i j)} k_{(j i)}\right)=\operatorname{det}\left(k_{(i j)}\right) \operatorname{tr}\left(k_{(i j)}^{-1}\right) \\
=\lambda_{1} \lambda_{2}+\lambda_{2} \lambda_{3}+\lambda_{3} \lambda_{1} \geqslant 0 \\
I_{3}=\epsilon_{i j k} k_{(1 j)} k_{(2 j)} k_{(3 j)}=\operatorname{det}\left(k_{(i j)}\right)=\lambda_{1} \lambda_{2} \lambda_{3} \geqslant 0
\end{gathered}
$$

While it is likely the case that Eqs. (14)-(16) could be inferred from [20], it is certainly not transparent. The conditions in [21] are more clear, but the necessary Eq. (16) is unaccountably omitted. This omission propagates to other sources, e.g., [25].

When the special case in which $\partial T / \partial x_{i}=(1,0,0)^{T}$ is substituted into Eq. (12), one finds that $k_{(11)} \geqslant 0$. Equivalent results are obtained for the remaining two directions. As the symmetric part of a diagonal element is the diagonal element itself, one then has

$$
k_{(11)}=k_{11} \geqslant 0, \quad k_{(22)}=k_{22} \geqslant 0, \quad k_{(33)}=k_{33} \geqslant 0
$$


It seems that no further significant simplifications can be made over those of Eqs. (14)-(17). However, it is useful to keep in mind the following imprecise, pragmatic, and rarely stated recipe: To insure positive definiteness of the conductivity tensor, terms on the diagonal must be positive and, relative to terms off the diagonal, large. In the two-dimensional limit, the conditions are simple and precise and reduce to $k_{(11)} \geqslant 0, k_{(22)} \geqslant 0$, and $\sqrt{k_{(11)} k_{(22)}}$ $\geqslant k_{(12)}$, or, equivalently, $k_{11} \geqslant 0, \quad k_{22} \geqslant 0$, and $\sqrt{k_{11} k_{22}} \geqslant\left(k_{12}\right.$ $\left.+k_{21}\right) / 2$.

Just as antisymmetric terms do not influence the entropy inequality, it is easy to show that they do not influence the energy equation. Substituting Eq. (10) into Eq. (1), one finds

$$
\rho \frac{\partial e}{\partial t}=k_{(i j)} \frac{\partial^{2} T}{\partial x_{i} \partial x_{j}}+\underbrace{k_{[i j]} \frac{\partial^{2} T}{\partial x_{i} \partial x_{j}}}_{=0}
$$

Once again the tensor inner product of the symmetric $\partial^{2} T / \partial x_{i} \partial x_{j}$ with the antisymmetric $k_{[i j]}$ identically vanishes, giving rise, after imposition of the caloric state equation (7), to a temperature evolution equation of

$$
\rho c \frac{\partial T}{\partial t}=k_{(i j)} \frac{\partial^{2} T}{\partial x_{i} \partial x_{j}}
$$

Now while an antisymmetric component of $k_{i j}$ cannot affect either the temperature field or the entropy inequality, it would induce a heat flux, and if such a flux existed, it could be measured with standard techniques. In fact in a hypothetical anisotropic material which had a purely antisymmetric thermal conductivity, one could imagine the following experiment. Take a thin rectangular plate and hold two parallel boundaries at two moderately different temperatures. The antisymmetric conductivity would then not induce a flux normal to the isothermal boundary, but would induce a parallel flux. This flux would induce as much energy to enter at one end and as to leave at the other. One could then put each of the nonisothermal boundaries in finite reservoirs of liquid water, and one would observe one reservoir solidify and the other boil. Such an observation has never been made, despite related careful attempts [4-6]. One might view this as a violation of the second law for the combined system of the reservoirs and the plate. If however, the reservoirs were infinite, the energy flux would induce negligible temperature change, and thus no violation of the second law.

In [9] it is contended that nonzero $k_{[i j]}$ can have no observable consequences. It is then argued that this implies that one can choose $k_{[i j]}$ arbitrarily without resort to Onsager reciprocity. Reference [9] goes on to choose $k_{[i j]}=0$, thus recovering Onsager reciprocity, while suggesting that other choices would be equally as valid. In light of the argument in the preceding paragraph, it is contended here that Onsager reciprocity

$$
k_{[i j]}=0
$$

is the unique choice to bring predictions of heat conduction in anisotropic materials into agreement with observation. This general conclusion is in agreement with Miller [11].

\section{Counter Examples}

Here three counterexamples are presented which illustrate the necessity of imposing the conditions described in the previous section.

Second Law Violation. Consider a conductivity tensor which is purely symmetric, as well as having positive diagonal elements, with a value of

$$
k_{i j}=k_{(i j)}=\left(\begin{array}{ccc}
1 & 1 & 2 \\
1 & 4 & -1 \\
2 & -1 & 5
\end{array}\right)
$$

It is easily seen that the two conditions of [21], Eqs. (14)-(15), are satisfied as $I_{1}=10$ and $I_{2}=23$. However, the tensor is not positive semi-definite since it has eigenvalues of $\lambda=6, \lambda=2$ $+\sqrt{5} \sim 4.2361$, and $\lambda=2-\sqrt{5} \sim-0.2361$. Here, the neglected $I_{3}$ $=-6$, and thus Eq. (16) is not satisfied, rendering the tensor not positive semi-definite, and the second law violated.

That this would lead to a nonphysical prediction is clearly seen upon rotating the coordinate system to one aligned with the principal axes of the conductivity tensor. The principal axes are given by the eigenvectors of $k_{(i j)}$. The normalized eigenvectors are then cast into the columns of an orthogonal matrix $\ell_{m n}$, which is the matrix of direction cosines of the rotation

$$
\ell_{m n}=\left(\begin{array}{ccc}
0.3015 & 0.3868 & -0.8715 \\
-0.3015 & 0.9058 & 0.2977 \\
0.9045 & 0.1730 & 0.3897
\end{array}\right)
$$

The transpose of this special matrix can be shown to be its inverse, and is also known as the rotation matrix, which results in $\ell_{j n} \ell_{m n}=\delta_{j m}$. Here, $\delta_{j m}$ is the Kronecker delta. As described in many sources, cf. [33], under rotation, a generic vector $v_{i}$ is represented in the rotated, denoted by a prime superscript, coordinate system as $v_{p}^{\prime}=\ell_{i p} v_{i}$. Likewise, a generic tensor $a_{i j}$ is represented as $a_{p n}^{\prime}=\ell_{i p} a_{i j} \ell_{j n}$. The relevant rotation operations are as follows:

$$
\begin{gathered}
q_{i}=-k_{i j} \frac{\partial T}{\partial x_{j}} \\
\ell_{i p} q_{i}=-\ell_{i p} k_{i j} \frac{\partial T}{\partial x_{j}} \\
\ell_{i p} q_{i}=-\ell_{i p} k_{i j} \delta_{j m} \frac{\partial T}{\partial x_{m}} \\
\ell_{i p} q_{i}=-\left(\ell_{i p} k_{i j} \ell_{j n}\right)\left(\ell_{m n} \frac{\partial T}{\partial x_{m}}\right) \\
q_{p}^{\prime}=-k_{p n}^{\prime} \frac{\partial T}{\partial x_{n}^{\prime}}
\end{gathered}
$$

The eigen-rotation of Eq. (22) renders $k_{p n}^{\prime}=\ell_{i p} k_{i j} \ell_{j n}$ to be a purely diagonal tensor with the eigenvalues on the diagonal. Consequently, the generalized Fourier's law in this particular reference frame is that of an orthotropic material and can be written as

$$
\left(\begin{array}{l}
q_{1}^{\prime} \\
q_{2}^{\prime} \\
q_{3}^{\prime}
\end{array}\right)=-\left(\begin{array}{ccc}
6 & 0 & 0 \\
0 & 4.2361 & 0 \\
0 & 0 & -0.2361
\end{array}\right)\left(\begin{array}{c}
\frac{\partial T}{\partial x_{1}^{\prime}} \\
\frac{\partial T}{\partial x_{2}^{\prime}} \\
\frac{\partial T}{\partial x_{3}^{\prime}}
\end{array}\right)
$$

Such a conductivity tensor admits the non-physical $q_{3}^{\prime}$ $=0.2361 \partial T / \partial x_{3}^{\prime}$; i.e., it predicts energy to be transported by random diffusion from regions of low temperature to regions of high temperature, in direct violation of Clausius' formulation of the second law.

Note that selection of a conductivity tensor which is more diagonally dominant can render the tensor to be positive semidefinite. For example, changing $k_{(11)}$ from 1 to 2 in Eq. (21) gives rise to satisfaction of the second law, as for this new tensor $\lambda_{1}$ $=6.1135, \lambda_{2}=0.4829$, and $\lambda_{3}=4.4036$. 
Onsager Reciprocity Violation, Plane Cartesian Case. Consider now a purely antisymmetric conductivity tensor applied to a strictly two-dimensional geometry. Take then

$$
k_{i j}=\left(\begin{array}{cc}
0 & -\kappa \\
\kappa & 0
\end{array}\right)
$$

where $\kappa$ is a characteristic scalar value of thermal conductivity. Obviously for this tensor $k_{(i j)}=0$ and $k_{[i j]}=k_{i j}$. From the generalized Fourier's law, Eq. (8), one then has

$$
\left(\begin{array}{l}
q_{1} \\
q_{2}
\end{array}\right)=-\left(\begin{array}{cc}
0 & -\kappa \\
\kappa & 0
\end{array}\right)\left(\begin{array}{c}
\frac{\partial T}{\partial x_{1}} \\
\frac{\partial T}{\partial x_{2}}
\end{array}\right)
$$

or more simply,

$$
q_{1}=\kappa \frac{\partial T}{\partial x_{2}}, \quad q_{2}=-\kappa \frac{\partial T}{\partial x_{1}}
$$

Substituting Eq. (26) into the Clausius-Duhem inequality, Eq. (6), the second law reduces to

$$
\frac{1}{T^{2}}\left(\begin{array}{ll}
\frac{\partial T}{\partial x_{1}} & \frac{\partial T}{\partial x_{2}}
\end{array}\right)\left(\begin{array}{cc}
0 & -\kappa \\
\kappa & 0
\end{array}\right)\left(\begin{array}{c}
\frac{\partial T}{\partial x_{1}} \\
\frac{\partial T}{\partial x_{2}}
\end{array}\right) \geqslant 0
$$

Expanding, one finds that the equality holds

$$
\frac{1}{T^{2}} \underbrace{\left.-\kappa \frac{\partial T}{\partial x_{1}} \frac{\partial T}{\partial x_{2}}+\kappa \frac{\partial T}{\partial x_{2}} \frac{\partial T}{\partial x_{1}}\right)}_{=0} \geqslant 0
$$

and consequently the irreversibility is identically zero for such a conductivity.

Similarly the first law of thermodynamics, Eq. (1), after application of the caloric state equation, Eq. (7), and Eq. (26), is written as

$$
\rho c \frac{\partial T}{\partial t}=\left(\frac{\partial}{\partial x_{1}} \frac{\partial}{\partial x_{2}}\right)\left(\begin{array}{cc}
0 & -\kappa \\
\kappa & 0
\end{array}\right)\left(\begin{array}{c}
\frac{\partial T}{\partial x_{1}} \\
\frac{\partial T}{\partial x_{2}}
\end{array}\right)
$$

This reduces to

$$
\rho c \frac{\partial T}{\partial t}=-\kappa \frac{\partial^{2} T}{\partial x_{1} \partial x_{2}}+\kappa \frac{\partial^{2} T}{\partial x_{2} \partial x_{1}}=0
$$

which holds that no temperature changes are induced by such a heat flux.

Consider now an initial boundary value problem for a plane Cartesian problem with a purely antisymmetric conductivity tensor. Consider the domain $x_{1} \in(-\infty, \infty), x_{2} \in[0, H], t \in[0, \infty)$, where $H$ is the height of the domain. Next, take as Dirichlet boundary conditions and initial conditions

$$
\begin{gathered}
T\left(x_{1}, 0, t\right)=T_{o}, \quad T\left(x_{1}, H, t\right)=T_{o}, \\
T\left(x_{1}, x_{2}, 0\right)=T_{o}+4\left(T_{\max }-T_{o}\right)\left(\frac{x_{2}}{H}\right)\left(1-\frac{x_{2}}{H}\right)
\end{gathered}
$$

Here, $T_{o}$ is the boundary temperature, and $T_{\max }$ is the maximum temperature, realized here at $x_{2}=H / 2$. Then Eq. (31) simply requires that the initial temperature distribution must hold for all time so that

$$
T\left(x_{1}, x_{2}, t\right)=T_{o}+4\left(T_{\max }-T_{o}\right)\left(\frac{x_{2}}{H}\right)\left(1-\frac{x_{2}}{H}\right)
$$

Application of Eq. (26) gives for the heat flux

$$
q_{1}\left(x_{1}, x_{2}, t\right)=\frac{4 \kappa\left(T_{\max }-T_{o}\right)}{H}\left(1-2 \frac{x_{2}}{H}\right), \quad q_{2}\left(x_{1}, x_{2}, t\right)=0
$$

The temperature gradient in the 2 direction induces a heat flux in the 1 direction. At the midplane, $x_{2}=H / 2$, there is no heat flux, and the maximum amplitude of the heat flux occurs at the boundaries with a magnitude of $4 \kappa\left(T_{\max }-T_{o}\right) / H$. In a rectangular control volume aligned with the domain, the same amount of energy enters at a given $x_{1}$ as exits at a downstream $x_{1}$. Moreover, all the energy remains confined to the domain. Consequently, the spatially nonuniform temperature field has no variation in time. Had the conduction tensor been that of an ordinary isotropic material, energy flux through the boundaries at $x_{2}=0$ and $x_{2}=H$ would have induced $T\left(x_{1}, x_{2}, t \rightarrow \infty\right)=0$. In the absence of a driving potential difference in temperature at the boundaries, a positive isotropic conductivity causes the system to come to equilibrium with its environment.

Onsager Reciprocity Violation: Plane Polar Case. A similar result is obtained in non-Cartesian coordinates. Consider a problem related to the previous example in plane polar coordinates, for which one has

$$
x_{1}=r \cos \theta, \quad x_{2}=r \sin \theta
$$

Application of standard coordinate transformations leads one to formulate the generalized Fourier's law for the antisymmetric conductivity tensor as

$$
q_{r}=\frac{\kappa}{r} \frac{\partial T}{\partial \theta}, \quad q_{\theta}=-\kappa \frac{\partial T}{\partial r}
$$

It is trivial to show that the first law still reduces to $\partial T / \partial t=0$, and that the second law is satisfied, with zero irreversibility. Consider now a similar initial boundary value problem on the domain of a circular disk for which $r \in[0, R], \theta \in[0,2 \pi], t \in[0, \infty)$ :

$$
\begin{gathered}
T(R, \theta, t)=T_{o}, \quad T(0, \theta, t)<\infty, \\
T(r, \theta, 0)=T_{o}+\left(T_{\max }-T_{o}\right)\left(1-\left(\frac{r}{R}\right)^{2}\right) .
\end{gathered}
$$

Here, $R$ is the outer radius of the disk. Once again the maximum temperature is $T_{\max }$, here realized at $r=0$. The solution for the time-dependent temperature field and heat flux is straightforwardly found to be

$$
\begin{gathered}
T(r, \theta, t)=T_{o}+\left(T_{\max }-T_{o}\right)\left(1-\left(\frac{r}{R}\right)^{2}\right) \\
q_{r}(r, \theta, t)=0 \\
q_{\theta}(r, \theta, t)=\frac{2 \kappa\left(T_{\max }-T_{o}\right)}{R}\left(\frac{r}{R}\right)
\end{gathered}
$$

Appropriately scaled temperature and heat flux fields are plotted in Fig. 1. Figure 1(a) shows the temperature field. Despite the lack of forcing either within the domain or at the boundary, the initial temperature disturbance persists for all time in the presence of purely antisymmetric conductivity. Figure 1(b) depicts the unusual consequence of a purely anti-symmetric conductivity: the heat flux vectors are tangent to the isotherms. This situation is analogous to velocity vectors and particle pathlines for a solid rotating about a central axis. In fact it is easy to show that the antisymmetric conductivity tensor has associated with it a vector which is analogous to the a solid body rotation vector; the equivalent vector here is $(1 / 2) \epsilon_{i j k} k_{[i j]}=(0,0, \kappa)^{T}$. Here, $\epsilon_{i j k}$ is the alternating unit tensor. Another analog is found in a classical chemical kinetics problem described by Onsager [1]. There it was shown that if Onsager reciprocity were not imposed on constitutive laws for chemical kinetics, that so-called cyclic reactions, never ob- 

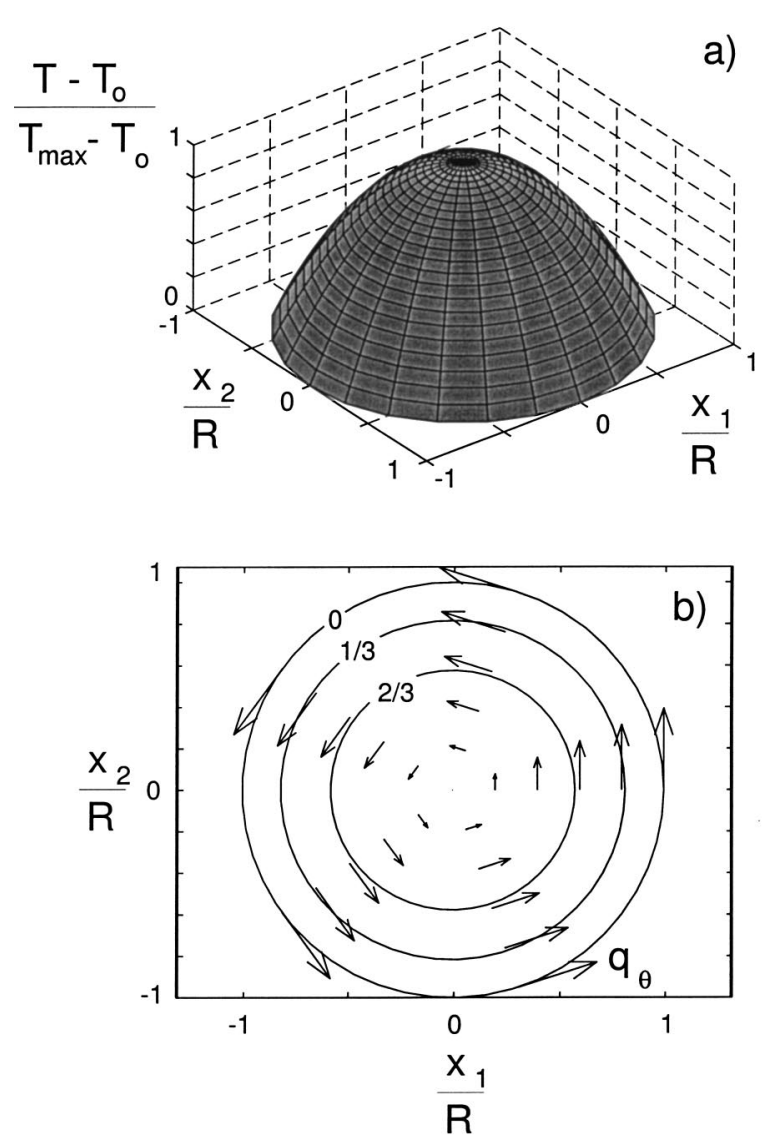

Fig. 1 Example solution for conductive heat transfer in an anisotropic material with a purely antisymmetric thermal conductivity tensor; (a) surface plot of temperature (dimensionless); and (b) isotherms of $\left(T-T_{o}\right) /\left(T_{\max }-T_{o}\right)$ and heat flux vectors (dimensionless)

served in closed adiabatic systems, could be predicted; for example, in a system with three components $A, B$, and $C$, one could predict a situation which at long time admitted the situation depicted in Fig. 2.

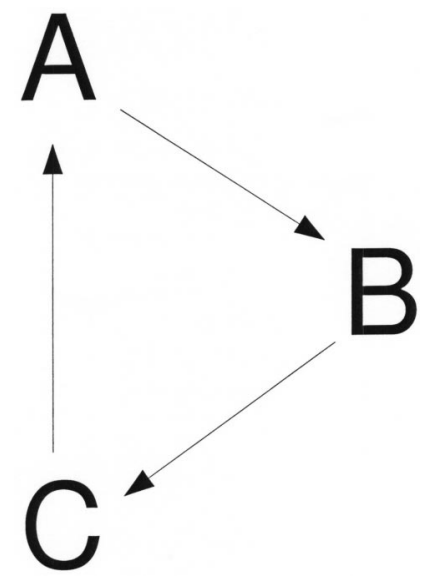

Fig. 2 Diagram of hypothesized cyclic chemical reaction for which Onsager reciprocity does not hold

\section{Conclusions}

As the scenario in the first example clearly violates the second law of thermodynamics, the necessity of the additional constraints for positive semi-definiteness is obvious. As the phenomenon predicted in the second two examples has never been observed in conductive heat transfer, despite the enforcement of first and second laws of thermodynamics, it is concluded that an additional condition is necessary. It is clear that Onsager reciprocity, equivalent to the Duhamel-Stokes conjecture, provides a sufficient condition.

Lastly, recent predictions such as those of [18] that Onsager reciprocity does not hold away from equilibrium suggest an interesting possibility. If this contention is correct, then it may be possible to repeat Soret's experiment [4], and with modern measurement devices, detect spiral components of heat fluxes in response to radial temperature gradients.

\section{Acknowledgment}

The author acknowledges the helpful comments of Prof. S. Paolucci of the University of Notre Dame.

\section{Nomenclature}

$A=$ generic chemical species

$B=$ generic chemical species

$C=$ generic chemical species

$H=$ domain height, [m]

$I_{1}=$ first invariant of conductivity tensor, $[\mathrm{W} / \mathrm{m} / \mathrm{K}]$

$I_{2}=$ second invariant of conductivity tensor, $\left[(\mathrm{W} / \mathrm{m} / \mathrm{K})^{2}\right]$

$I_{3}=$ third invariant of conductivity tensor, $\left[(\mathrm{W} / \mathrm{m} / \mathrm{K})^{3}\right]$

$R=$ domain outer radius, $[\mathrm{m}]$

$T=$ temperature, $[\mathrm{K}]$

$a_{i j}=$ generic tensor

$c=$ specific heat, $[\mathrm{J} / \mathrm{kg} / \mathrm{K}]$

$e=$ specific internal energy, $[\mathrm{J} / \mathrm{kg}]$

$k_{i j}=$ thermal conductivity tensor, $[\mathrm{W} / \mathrm{m} / \mathrm{K}]$

$\ell_{i j}=$ direction cosine tensor

$q_{i}=$ heat flux vector, $\left[\mathrm{W} / \mathrm{m}^{2}\right]$

$r=$ radial coordinate, $[\mathrm{m}]$

$s=$ specific entropy, $[\mathrm{J} / \mathrm{kg} / \mathrm{K}]$

$t=$ time, $[\mathrm{s}]$

$v_{i}=$ generic vector

$x_{i}=$ Cartesian distance vector coordinate, $[\mathrm{m}]$

\section{Greek Symbols}

$\delta_{j m}=$ Kronecker delta

$\epsilon_{i j k}=$ alternating unit tensor

$\theta=$ circumferential coordinate

$\kappa=$ thermal conductivity scalar component, $[\mathrm{W} / \mathrm{m} / \mathrm{K}]$

$\lambda=$ eigenvalue of conductivity tensor, $[\mathrm{W} / \mathrm{m} / \mathrm{K}]$

$\rho=$ density, $\left[\mathrm{kg} / \mathrm{m}^{3}\right]$

Subscripts

$$
\begin{aligned}
& i=\text { Cartesian index } \\
& j=\text { Cartesian index }
\end{aligned}
$$

$m=$ Cartesian index

$\max =$ maximum

$n=$ Cartesian index

$o=$ reference state

$p=$ Cartesian index

$r=$ radial direction

$\theta=$ circumferential direction

$(\cdots)=$ symmetric part of tensor

$[\cdots]=$ antisymmetric part of tensor

Superscript

$'$ = rotated coordinate system

\section{References}

[1] Onsager, L., 1931, "Reciprocal Relations in Irreversible Processes. I," Phys. Rev., 37(4), pp. 405-426. 
[2] Duhamel, J.-M.-C., 1832, “Sur les Équations Générales de la Propagation de la Chaleur dans les Corps Solides dont la Conductibilité n'est pas la Même dans Tous les Sens," J. Ec. Polytech. (Paris), 13(21), pp. 356-399.

[3] Stokes, G. G., 1851, "On the Conduction of Heat in Crystals," Cambridge and Dublin Math. J., 6, pp. 215-238.

[4] Soret, C., 1893, "Sur l'Étude Expérimentale des Coefficients Rotationnels de Conductibilité Thermique,” Arch. Sci. Phys. Nat., 29, pp. 355-357.

[5] Voigt, W., 1903, "Fragen der Kristallphysik I. Über die Rotatorischen Constanten der Wärmeleitung von Apatit und Dolomit," Nachr. Ges. Wiss. Goettingen, Math.-Phys. Kl., 3, pp. 87-89.

[6] Voigt, W., 1910, Lehrbuch der Kristallphysik, Teubner, Leipzig.

[7] Müller, I., and Ruggeri, T., 1998, Rational Extended Thermodynamics, Second ed., Springer, New York.

[8] deGroot, S. R., and Masur, P., 1984, Non-Equilibrium Thermodynamics, Dover, New York.

[9] Casimir, H. B. G., 1945, “On Onsager's Principle of Microscopic Reversibility," Rev. Mod. Phys., 17(2-3), pp. 343-350.

[10] Prigogene, I., 1967, Thermodynamics of Irreversible Processes, Third ed., Wiley, New York.

[11] Miller, D., 1960, "Thermodynamics of Irreversible Processes, the Experimental Verification of the Onsager Reciprocal Relations," Chem. Rev. (Washington, D.C.), 60, pp. 15-37.

[12] Woods, L. C., 1975, The Thermodynamics of Fluid Systems, Clarendon, Oxford.

[13] Fung, Y. C., 1965, Foundations of Solid Mechanics, Prentice-Hall, Englewood Cliffs, New Jersey.

[14] Truesdell, C., 1969, Rational Thermodynamics, McGraw-Hill, New York.

[15] Day, W. A., and Gurtin, M. E., 1969, "On the Symmetries of the Conductivity Tensor and Other Restrictions in the Nonlinear Theory of Heat Conduction," Arch. Ration. Mech. Anal., 33, pp. 26-32.

[16] Oono, Y., 1993, "Onsager's Principle From Large Deviation Point of View," Prog. Theor. Phys., 89(5), pp. 973-983.

[17] Sieniutycz, S., and Berry, R. S., 1993, “Canonical Formalism, Fundamental Equation, and Generalized Thermomechanics for Irreversible Fluids With Heat Transfer," Phys. Rev. E, 47(3), pp. 1765-1783.

[18] Allahverdyan, A. E., and Nieuwenhuizen, Th. M., 2000, "Adiabatic Steady State: Its Thermodynamics, Entropy Production, Energy Dissipation, and Vio- lation of Onsager Relations," Phys. Rev. E, 62(1), pp. 845-850.

[19] LaCour, B. R., and Schieve, W. C., 2004, "Derivation of the Onsager Principle From Large Deviation Theory," Physica A, 331(1-2), pp. 109-124.

[20] Carslaw, H. S., and Jaeger, J. C., 1986, Conduction of Heat in Solids, Second ed., Clarendon, Oxford.

[21] Özişik, M. N., 1993, Heat Conduction, Wiley, New York.

[22] Bird, R. B., Stewart, W. E., and Lightfoot, E. N., 1960, Transport Phenomena, Wiley, New York.

[23] Gebhardt, B., 1993, Heat Conduction and Mass Diffusion, McGraw-Hill, New York.

[24] Mulholland, G. P., and Gupta, B. P., 1977, "Heat Transfer in a ThreeDimensional Anisotropic Solid of Arbitrary Shape," ASME J. Heat Transfer, 99(1), pp. 135-137.

[25] Chang, Y. P., 1977, "Analytical Solution for Heat Conduction in Anisotropic Media in Infinite, Semi-Infinite, and Two-Plane-Bounded Regions," Int. J. Heat Mass Transfer, 20(10), pp. 1019-1028.

[26] Sadd, M. H., and Miskioglu, I., 1978, "Temperatures in an Anisotropic Sheet Containing an Insulated Elliptical Hole," ASME J. Heat Transfer, 100(3), pp. 553-555.

[27] Chang, Y. P., and Poon, K. C., 1979, "Three-Dimensional, Steady-State HeatConduction in Cylinders of General Anisotropic-Media," ASME J. Heat Transfer, 101(3), pp. 548-553.

[28] Huang, S. C., and Chang, Y. P., 1984, "Anisotropic Heat-Conduction With Mixed Boundary Conditions," ASME J. Heat Transfer, 106(3), pp. 646-648.

[29] Zhang, X. Z., 1990, "Steady-State Temperatures in an Anisotropic Strip," ASME J. Heat Transfer, 112(1), pp. 16-20.

[30] Hsieh, M.-H., and Ma, C.-C., 2002, "Analytical Investigations for Heat Conduction Problems in Anisotropic Thin-Layer Media With Embedded Heat Sources," Int. J. Heat Mass Transfer, 45(20), pp. 4117-4132.

[31] Ma, C.-C., and Chang, S.-W., 2004, "Analytical Exact Solutions of Heat Conduction Problems for Anisotropic Multi-Layered Media," Int. J. Heat Mass Transfer, 47(8-9), pp. 1643-1655.

[32] Strang, G., 1988, Linear Algebra and its Application, Third ed., Harcourt Brace Jovanovich, Fort Worth, TX.

[33] Aris, R., 1962, Vectors, Tensors, and the Basic Equations of Fluid Mechanics, Dover, New York. 